Proyecciones Journal of Mathematics

Vol. 38, No 1, pp. 97-110, March 2019.

Universidad Católica del Norte

Antofagasta - Chile

\title{
Asymptotic behavior of linear advanced dynamic equations on time scales
}

\author{
Malik Belaid \\ University of Annaba, Algeria \\ Abdelouaheb Ardjouni \\ University of Souk Ahras, Algeria \\ and \\ Ahcene Djoudi \\ University of Annaba, Algeria \\ Received : January 2018. Accepted : October 2018
}

\begin{abstract}
Let $\mathbf{T}$ be a time scale which is unbounded above and below and such that $t_{0} \in \mathbf{T}$. Let $i d+h, i d+r:\left[t_{0}, \infty\right) \cap \mathbf{T} \rightarrow \mathbf{T}$ be such that $(i d+h)\left(\left[t_{0}, \infty\right) \cap \mathbf{T}\right)$ and $(i d+r)\left(\left[t_{0}, \infty\right) \cap \mathbf{T}\right)$ are time scales. We use the contraction mapping theorem to obtain convergence to zero about the solution for the following linear advanced dynamic equation

$x^{\triangle}(t)+a(t) x^{\sigma}(t+h(t))+b(t) x^{\sigma}(t+r(t))=0, t \in\left[t_{0}, \infty\right) \cap \mathbf{T}$,

where $f^{\triangle}$ is the $\triangle$-derivative on $\mathbf{T}$. A convergence theorem with a necessary and sufficient condition is proved. The results obtained here extend the work of Dung [11]. In addition, the case of the equation with several terms is studied.
\end{abstract}

Subjclass [2010] : 34K20, 34K30, 34k40.

Keywords : Fixed points, advanced dynamic equations, Asymptotic behavior, time scales. 


\section{Introduction}

The concept of time scales analysis is a fairly new idea. In 1988, it was introduced by the German mathematician Stefan Hilger in his Ph.D. thesis [13]. It combines the traditional areas of continuous and discrete analysis into one theory. After the publication of two textbooks in this area by Bohner and Peterson [6] and [7], more and more researchers were getting involved in this fast-growing field of mathematics.

The study of dynamic equations brings together the traditional research areas of differential and difference equations. It allows one to handle these two research areas at the same time, hence shedding light on the reasons for their seeming discrepancies. In fact, many new results for the continuous and discrete cases have been obtained by studying the more general time scales case (see $[1,3,4,14]$ and the references therein).

There is no doubt that the Lyapunov method have been used successfully to investigate stability properties of wide variety of ordinary, functional and partial equations. Nevertheless, the application of this method to problem of stability in differential equations with delay has encountered serious difficulties if the delay is unbounded or if the equation has unbounded term. It has been noticed that some of theses difficulties vanish by using the fixed point technic. Other advantages of fixed point theory over Lyapunov's method is that the conditions of the former are average while those of the latter are pointwise (see $[2,5,8,9,10,11,12]$ and references therein).

In paper, we consider the following linear advanced dynamic equation

$x^{\triangle}(t)+a(t) x^{\sigma}(t+h(t))+b(t) x^{\sigma}(t+r(t))=0, t \in\left[t_{0}, \infty\right) \cap \mathbf{T}$,

where $\mathbf{T}$ is an unbounded above and below time scale and such that $t_{0} \in \mathbf{T}$, $a$ and $b$ are rd-continuous functions on $\left[t_{0}, \infty\right) \cap \mathbf{T}$, the advanced arguments $h$ and $r$ are rd-continuous functions with $h(t) \geq 0$ and $r(t) \geq 0$. We assume that $(i d+h)\left(\left[t_{0}, \infty\right) \cap \mathbf{T}\right)$ and $(i d+r)\left(\left[t_{0}, \infty\right) \cap \mathbf{T}\right)$ are time scales where $i d$ is the identity function.

Our purpose here is to use the contraction mapping theorem (see [15]) to show the convergence to zero about the solution for (1.1). A convergence theorem with a necessary and sufficient condition is proved. In the special case $\mathbf{T}=\mathbf{R}$, Dung [11] shows the solution of (1.1) is convergent to zero with a necessary and sufficient condition by using the contraction mapping theorem. 
In Section 2, we present some preliminary material that we will need through the remainder of the paper. We will state some facts about the exponential function on a time scale. We present our main results on convergence in Section 3. A study of the general form of (1.1) with several terms is also given. The results presented in this paper extend the main results in [11].

\section{Preliminaries}

In this section, we consider some advanced topics in the theory of dynamic equations on a time scales. Again, we remind that for a review of this topic we direct the reader to the monographs of Bohner and Peterson [6] and [7].

A time scale $\mathbf{T}$ is a closed nonempty subset of $\mathbf{R}$. For $t \in \mathbf{T}$ the forward jump operator $\sigma$ is defined as $\sigma(t)=\inf \{s \in \mathbf{T}: s>t\}$. This operator allow elements in the time scale to be classified as follows. We say $t$ is right scattered if $\sigma(t)>t$ and right dense if $\sigma(t)=t$. The graininess function $\mu: \mathbf{T} \rightarrow[0, \infty)$ is defined by $\mu(t)=\sigma(t)-t$ and gives the distance between an element and its successor. We set $\inf \emptyset=\sup \mathbf{T}$ and $\sup \emptyset=\inf \mathbf{T}$. If $\mathbf{T}$ has a left scattered maximum $M$, we define $\mathbf{T}^{k}=\mathbf{T} \backslash\{M\}$. Otherwise, we define $\mathbf{T}^{k}=\mathbf{T}$. If $\mathbf{T}$ has a right scattered minimum $m$, we define $\mathbf{T}_{k}=\mathbf{T} \backslash\{m\}$. Otherwise, we define $\mathbf{T}_{k}=\mathbf{T}$.

Let $t \in \mathbf{T}^{k}$ and let $f: \mathbf{T} \rightarrow \mathbf{R}$. The delta derivative of $f(t)$, denoted $f^{\triangle}(t)$, is defined to be the number (when it exists), with the property that, for each $\epsilon>0$, there is a neighborhood $U$ of $t$ such that

$$
\left|f(\sigma(t))-f(s)-f^{\triangle}(t)[\sigma(t)-s]\right| \leq \epsilon|\sigma(t)-s|,
$$

for all $s \in U$. If $\mathbf{T}=\mathbf{R}$ then $f^{\triangle}(t)=f^{\prime}(t)$ is the usual derivative. If $\mathbf{T}=\mathbf{Z}$ then $f^{\triangle}(t)=\triangle f(t)=f(t+1)-f(t)$ is the forward difference of $f$ at $t$.

A function $f$ is right dense continuous (rd-continuous), $f \in C_{r d}=$ $C_{r d}(\mathbf{T}, \mathbf{R})$, if it is continuous at every right dense point $t \in \mathbf{T}$ and its lefthand limits exist at each left dense point $t \in \mathbf{T}$. The function $f: \mathbf{T} \rightarrow \mathbf{R}$ is differentiable on $\mathbf{T}^{k}$ provided $f^{\triangle}(t)$ exists for all $t \in \mathbf{T}^{k}$.

We are now ready to state some properties of the delta-derivative of $f$. Note $f^{\sigma}(t)=f(\sigma(t))$.

Theorem 1 ([6, Theorem 1.20]). Assume $f, g: \mathbf{T} \rightarrow \mathbf{R}$ are differentiable at $t \in \mathbf{T}^{k}$ and let $\alpha$ be a scalar. $(i)(f+g)^{\triangle}(t)=g^{\triangle}(t)+f^{\triangle}(t)$. (ii) $(\alpha f)^{\triangle}(t)=\alpha f^{\triangle}(t)$. (iii) The product rules 


$$
\begin{aligned}
& (f g)^{\triangle}(t)=f^{\triangle}(t) g(t)+f^{\sigma}(t) g^{\triangle}(t), \\
& (f g)^{\triangle}(t)=f(t) g^{\triangle}(t)+f^{\triangle}(t) g^{\sigma}(t) .
\end{aligned}
$$

(iv) If $g(t) g^{\sigma}(t) \neq 0$ then

$$
\left(\frac{f}{g}\right)^{\triangle}(t)=\frac{f^{\triangle}(t) g(t)-f(t) g^{\triangle}(t)}{g(t) g^{\sigma}(t)} .
$$

The next theorem is the chain rule on time scales ([6, Theorem 1.93], Theorem 1.93).

Theorem 2 (Chain Rule). Assume $\nu: \mathbf{T} \rightarrow \mathbf{R}$ is strictly increasing and $\widetilde{\mathbf{T}}:=\nu(\mathbf{T})$ is a time scale. Let $\omega: \widetilde{\mathbf{T}} \rightarrow \mathbf{R}$. If $\nu^{\triangle}(t)$ and $\omega^{\widetilde{\triangle}}(\nu(t))$ exist for $t \in \mathbf{T}^{k}$, then $(\omega \circ \nu)^{\triangle}=\left(\omega^{\widetilde{\triangle}} \circ \nu\right) \nu^{\triangle}$.

A function $F: \mathbf{T} \rightarrow \mathbf{R}$ is said to be a delta antiderivative of $f: \mathbf{T} \rightarrow \mathbf{R}$, provided $F^{\triangle}(t)=f(t)$ for all $t \in \mathbf{T}^{k}$. For all $a, b \in \mathbf{T}, a<b$, the delta integral of $f$ from $a$ to $b$ is defined by

$$
\int_{a}^{b} f(t) \triangle t=F(b)-F(a) .
$$

In the sequel we will need to differentiate and integrate functions of the form $f(t-\tau(t))=f(\nu(t))$ where, $\nu(t):=t-\tau(t)$. Our next theorem is the substitution rule ([6, Theorem 1.98], Theorem 1.98).

Theorem 3 (Substitution). Assume $\nu: \mathbf{T} \rightarrow \mathbf{R}$ is strictly increasing and $\widetilde{\mathbf{T}}:=\nu(T)$ is a time scale. If $f: \mathbf{T} \rightarrow \mathbf{R}$ is rd-continuous function and $\nu$ is differentiable with rd-continuous derivative, then for $a, b \in T$,

$$
\int_{a}^{b} f(t) \nu^{\triangle}(t) \triangle t=\int_{\nu(a)}^{\nu(b)}\left(f \circ \nu^{-1}\right)(s) \widetilde{\triangle} s .
$$

A function $p: \mathbf{T} \rightarrow \mathbf{R}$ is said to be regressive provided $1+\mu(t) p(t) \neq 0$ for all $t \in \mathbf{T}^{k}$. The set of all regressive rd-continuous function $f: \mathbf{T} \rightarrow \mathbf{R}$ is denoted by $\mathcal{R}$. The set of all positively regressive functions $\mathcal{R}^{+}$, is given by $\mathcal{R}^{+}=\{f \in \mathcal{R}: 1+\mu(t) f(t)>0$ for all $t \in \mathbf{T}\}$.

Let $p \in \mathcal{R}$ and $\mu(t) \neq 0$ for all $t \in \mathbf{T}$. The exponential function on $\mathbf{T}$ is defined by 


$$
e_{p}(t, s)=\exp \left(\int_{s}^{t} \frac{1}{\mu(z)} \log (1+\mu(z) p(z)) \Delta z\right) .
$$

It is well known that if $p \in \mathcal{R}^{+}$, then $e_{p}(t, s)>0$ for all $t \in \mathbf{T}$. Also, the exponential function $y(t)=e_{p}(t, s)$ is the solution to the initial value problem $y^{\triangle}=p(t) y, y(s)=1$. Other properties of the exponential function are given by the following lemma.

Lemma 1 ([6, Theorem 2.36]). Let $p, q \in \mathcal{R}$. Then

(i) $e_{0}(t, s)=1$ and $e_{p}(t, t)=1$,

(ii) $e_{p}(\sigma(t), s)=(1+\mu(t) p(t)) e_{p}(t, s)$,

(iii) $\frac{1}{e_{p}(t, s)}=e_{\ominus p}(t, s)$, where $\ominus p(t)=-\frac{p(t)}{1+\mu(t) p(t)}$,

(iv) $e_{p}(t, s)=\frac{1}{e_{p}(s, t)}=e_{\ominus p}(s, t)$,

(v) $e_{p}(t, s) e_{p}(s, r)=e_{p}(t, r)$,

(vi) $e_{p}^{\triangle}(., s)=p e_{p}(., s)$ and $\left(\frac{1}{e_{p}(., s)}\right)^{\triangle}=-\frac{p(t)}{e_{p}^{\sigma}(., s)}$.

Lemma 2 ([1]). If $p \in \mathcal{R}^{+}$, then

$$
0<e_{p}(t, s) \leq \exp \left(\int_{s}^{t} p(u) \triangle u\right), \forall t \in \mathbf{T} .
$$

\section{Main Results}

In this section, we state and prove our main results. Before doing these, let us recall a definition of the solution to (1.1).

Definition 1. A rd-continuously differentiable function $x:\left[t_{0}, \infty\right) \cap \mathbf{T} \rightarrow$ $\mathbf{R}$ is called a solution of equation (1.1), if it satisfies the relation (1.1) for all $t \in\left[t_{0}, \infty\right) \cap \mathbf{T}$.

We need a technical lemma which plays a key role in this article. This lemma transforms (1.1) into an equivalent integral equation for which the method of fixed points can work.

Lemma 3. Let $x$ be the solution of (1.1) on $\left[t_{0}, \infty\right) \cap \mathbf{T}$. Then, $x$ satisfies the following integral equation

$$
\begin{aligned}
x(t) & =x_{0} e_{\ominus D}\left(t, t_{0}\right)+\int_{t_{0}}^{t} e_{\ominus D}(t, s) a(s)\left(\int_{\sigma(s)}^{\sigma(s+h(s))} E_{x}(u) \Delta u\right) \Delta s \\
& +\int_{t_{0}}^{t} e_{\ominus D}(t, s) b(s)\left(\int_{\sigma(s)}^{\sigma(s+r(s))} E_{x}(u) \Delta u\right) \Delta s, t \in\left[t_{0}, \infty\right) \cap \mathbf{T},
\end{aligned}
$$

where $x_{0}=x\left(t_{0}\right), D(t)=a(t)+b(t)$ and $E_{x}(t)=a(t) x^{\sigma}(t+h(t))+$ $b(t) x^{\sigma}(t+r(t))$. 
Proof. Using the relation

$$
x^{\sigma}(u)-x^{\sigma}(t)=\int_{\sigma(t)}^{\sigma(u)} x^{\triangle}(s) \Delta s,
$$

we can rewrite equation (1.1) as follows,

$$
\begin{gathered}
x^{\triangle}(t)=-[a(t)+b(t)] x^{\sigma}(t)-a(t) \int_{\sigma(t)}^{\sigma(t+h(t))} x^{\triangle}(s) \Delta s \\
-b(t) \int_{\sigma(t)}^{\sigma(t+r(t))} x^{\triangle}(s) \Delta s .
\end{gathered}
$$

After substituting $x^{\triangle}$ from (1.1), we obtain

$$
\begin{aligned}
& x^{\triangle}(t)=-[a(t)+b(t)] x^{\sigma}(t)+a(t) \int_{\sigma(t)}^{\sigma(t+h(t))}\left(a(s) x^{\sigma}(s+h(s))\right. \\
& \left.\quad+b(s) x^{\sigma}(s+r(s))\right) \Delta s+b(t) \int_{\sigma(t)}^{\sigma(t+r(t))}\left(a(s) x^{\sigma}(s+h(s))\right. \\
& \left.\quad+b(s) x^{\sigma}(s+r(s))\right) \Delta s,
\end{aligned}
$$

or equivalently,

$$
x^{\triangle}(t)+D(t) x^{\sigma}(t)=a(t) \int_{\sigma(t)}^{\sigma(t+h(t))} E_{x}(s) \Delta s+b(t) \int_{\sigma(t)}^{\sigma(t+r(t))} E_{x}(s) \Delta s .
$$

Multiplying both sides of (3.2) by the factor $e_{D}\left(t, t_{0}\right)$ and then integration from $t_{0}$ to $t$, we obtain

$$
\begin{aligned}
x(t) e_{D}\left(t, t_{0}\right)-x\left(t_{0}\right) & =\int_{t_{0}}^{t} e_{D}\left(s, t_{0}\right) a(s)\left(\int_{\sigma(s)}^{\sigma(s+h(s))} E_{x}(u) \Delta u\right) \Delta s \\
& +\int_{t_{0}}^{t} e_{D}\left(s, t_{0}\right) b(s)\left(\int_{\sigma(s)}^{\sigma(s+r(s))} E_{x}(u) \Delta u\right) \Delta s,
\end{aligned}
$$

which means that $x$ is the solution of (3). The lemma is proved.

Theorem 4. Assume that the following conditions hold,

$$
\begin{gathered}
D \in \mathcal{R}^{+}, \lim _{t \rightarrow \infty} \int_{t_{0}}^{t} \frac{1}{\mu(\tau)} \log (1+\mu(\tau) D(\tau)) \Delta \tau=\infty \\
\sup _{t \geq t_{0}} \int_{t_{0}}^{t} e_{\ominus D}\left(s, t_{0}\right)\left(|a(s)| \int_{\sigma(s)}^{\sigma(s+h(s))}(|a(u)|+|b(u)|) \Delta u\right. \\
\left.+|b(s)| \int_{\sigma(s)}^{\sigma(s+r(s))}(|a(u)|+|b(u)|) \Delta u\right) \Delta s=\alpha<1 .
\end{gathered}
$$

Then, any solution $x$ of (1.1) converges to zero, that is, $\lim _{t \rightarrow \infty} x(t)=0$. 
Proof. Let $x$ be an arbitrary solution (1.1). We then can define $x_{0}:=$ $x\left(t_{0}\right)$. Thanks to Lemma 3 , we know that $x$ is a solution of equation (3) with a initial condition $x\left(t_{0}\right)=x_{0}$. As a consequence, in order to obtain the desired result, it is enough to show that equation (3) with the initial condition $x\left(t_{0}\right)=x_{0}$ has an unique solution and this solution converges to zero as $t$ tends to $\infty$.

Denote by $\mathbf{C}$ the space of bounded continuous functions $x$ on $\left[t_{0}, \infty\right) \cap \mathbf{T}$ such that $x\left(t_{0}\right)=x_{0}$. It is seen that $\mathbf{C}$ is a complete metric space with metric

$$
\rho(x, y)=\sup _{t \geq t_{0}}|x(t)-y(t)| .
$$

We define the operator $\mathbf{P}$ on $\mathbf{C}$ as

$$
\begin{aligned}
& (\mathbf{P} x)(t)=x_{0} e_{\ominus D}\left(t, t_{0}\right)+\int_{t_{0}}^{t} e_{\ominus D}(t, s) a(s)\left(\int_{\sigma(s)}^{\sigma(s+h(s))} E_{x}(u) \Delta u\right) \Delta s \\
& +\int_{t_{0}}^{t} e_{\ominus D}(t, s) b(s)\left(\int_{\sigma(s)}^{\sigma(s+r(s))} E_{x}(u) \Delta u\right) \Delta s, t \in\left[t_{0}, \infty\right) \cap \mathbf{T} .
\end{aligned}
$$

Obviously, we have $\mathbf{P}(\mathbf{C}) \subset \mathbf{C}$. Let $x, y \in \mathbf{C}$, then $x\left(t_{0}\right)=y\left(t_{0}\right)=x_{0}$ and hence, we have

$$
\begin{aligned}
& |(\mathbf{P} x)(t)-(\mathbf{P} y)(t)| \\
\leq & \int_{t_{0}}^{t} e_{\ominus D}(t, s)|a(s)|\left(\int_{\sigma(s)}^{\sigma(s+h(s))}\left|E_{x}(u)-E_{y}(u)\right| \Delta u\right) \Delta s \\
+ & \int_{t_{0}}^{t} e_{\ominus D}(t, s)|b(s)|\left(\int_{\sigma(s)}^{\sigma(s+r(s))}\left|E_{x}(u)-E_{y}(u)\right| \Delta u\right) \Delta s, t \in\left[t_{0}, \infty\right) \cap \mathbf{T},
\end{aligned}
$$

where

$$
\begin{aligned}
& \left|E_{x}(u)-E_{y}(u)\right| \leq|a(u)|\left|x^{\sigma}(u+h(u))-y^{\sigma}(u+h(u))\right| \\
+ & |b(u)|\left|x^{\sigma}(u+r(u))-y^{\sigma}(u+r(u))\right| \\
\leq & (|a(u)|+|b(u)|) \rho(x, y) .
\end{aligned}
$$

As a consequence, we have

$$
\begin{aligned}
& |(\mathbf{P} x)(t)-(\mathbf{P} y)(t)| \\
\leq & {\left[\int _ { t _ { 0 } } ^ { t } e _ { \ominus D } ( t , s ) \left(|a(s)| \int_{\sigma(s)}^{\sigma(s+h(s))}(|a(u)|+|b(u)|) \Delta u\right.\right.} \\
+ & \left.\left.|b(s)| \int_{\sigma(s)}^{\sigma(s+r(s))}(|a(u)|+|b(u)|) \Delta u\right) \Delta s\right] \rho(x, y), t \in\left[t_{0}, \infty\right) \cap \mathbf{T} .
\end{aligned}
$$

This combine with (3) yields

$$
\rho(\mathbf{P} x, \mathbf{P} y) \leq \alpha \rho(x, y) .
$$

As $\alpha<1$, we can conclude that $\mathbf{P}$ is a contraction operator. 
We now consider a closed subspace $\mathbf{S}$ of $\mathbf{C}$

$$
\mathbf{S}=\left\{x \in \mathbf{C}: \lim _{t \rightarrow \infty} x(t)=0\right\}
$$

We claim that $\mathbf{P}(\mathbf{S}) \subset \mathbf{S}$. Indeed, let $x \in \mathbf{S}$, then we have

$$
\begin{aligned}
& |(\mathbf{P} x)(t)| \leq\left|x_{0}\right| e_{\ominus D}\left(t, t_{0}\right)+\int_{t_{0}}^{t} e_{\ominus D}(t, s)|a(s)|\left(\int_{\sigma(s)}^{\sigma(s+h(s))}\left|E_{x}(u)\right| \Delta u\right) \Delta s \\
+ & \int_{t_{0}}^{t} e_{\ominus D}(t, s)|b(s)|\left(\int_{\sigma(s)}^{\sigma(s+r(s))}\left|E_{x}(u)\right| \Delta u\right) \Delta s \\
:= & I_{1}+I_{2}+I_{3}, t \in\left[t_{0}, \infty\right) \cap \mathbf{T},
\end{aligned}
$$

where

$$
I_{1}=\left|x_{0}\right| e_{\ominus D}\left(t, t_{0}\right), I_{2}=\int_{t_{0}}^{t} e_{\ominus D}(t, s)|a(s)|\left(\int_{\sigma(s)}^{\sigma(s+h(s))}\left|E_{x}(u)\right| \Delta u\right) \Delta s,
$$

and

$$
I_{3}=\int_{t_{0}}^{t} e_{\ominus D}(t, s)|b(s)|\left(\int_{\sigma(s)}^{\sigma(s+r(s))}\left|E_{x}(u)\right| \Delta u\right) \Delta s .
$$

By (3.3) we obtain $I_{1} \rightarrow 0$ as $t \rightarrow \infty$. Moreover, it follows from the fact $x \in \mathbf{S}$ that for any $\varepsilon>0$, there exists $T \geq t_{0}$ such that $|x(t)|<\frac{\varepsilon}{2}$ for all $t \geq T$. Hence, we have

$$
\begin{aligned}
& \mathrm{I}_{2}=\int_{t_{0}}^{T} e_{\ominus D}(t, s)|a(s)|\left(\int_{\sigma(s)}^{\sigma(s+h(s))}\left|E_{x}(u)\right| \Delta u\right) \Delta s \\
+ & \int_{T}^{t} e_{\ominus D}(t, s)|a(s)|\left(\int_{\sigma(s)}^{\sigma(s+h(s))}\left|E_{x}(u)\right| \Delta u\right) \Delta s \\
\leq & \int_{t_{0}}^{T} e_{\ominus D}(t, s)|a(s)|\left(\int_{\sigma(s)}^{\sigma(s+h(s))}\left|E_{x}(u)\right| \Delta u\right) \Delta s \\
+ & \frac{\varepsilon}{2} \int_{T}^{t} e_{\ominus D}(t, s)|a(s)|\left(\int_{\sigma(s)}^{\sigma(s+h(s))}(|a(u)|+|b(u)|) \Delta u\right) \Delta s, t \geq T .
\end{aligned}
$$

We observe that the first term in the right hand side of (3) converges to zero as $t \rightarrow \infty$ due to condition (3.3). Thus, there exists $T_{1} \geq T$, such that

$I_{2} \leq \frac{\varepsilon}{2}+\frac{\varepsilon}{2} \int_{T}^{t} e_{\ominus D}(t, s)|a(s)|\left(\int_{\sigma(s)}^{\sigma(s+h(s))}(|a(u)|+|b(u)|) \Delta u\right) \Delta s, t \geq T_{1}$.

Using (3) we get $I_{2}<\varepsilon$ for all $t \geq T_{1}$. In other words, we have $I_{2} \rightarrow 0$ as $t \rightarrow \infty$. Similarly, we also have $I_{3} \rightarrow 0$ as $t \rightarrow \infty$. Hence, $(\mathbf{P} x)(t) \rightarrow 0$ as $t \rightarrow \infty$. 
In summary, $\mathbf{P}$ is a contraction operator and $\mathbf{P}(\mathbf{S}) \subset \mathbf{S}$. By the contraction mapping principle, $\mathbf{P}$ has a unique fixed point $x$ in $\mathbf{S}$, that is,

$$
x=\mathbf{P} x \text { and } \lim _{t \rightarrow \infty} x(t)=0 .
$$

This means that equation (3) has a unique solution and this solution satisfies $\lim _{t \rightarrow \infty}$. The proof is complete.

Example 1. Let $\mathbf{T}=\mathbf{R}$. Consider the equation

$x^{\prime}(t)+0.2 \cos (t) x(t+0.5)+0.05(1+\sin (t)) x\left(t+\cos ^{2}(t)\right)=0, t \geq 0$.

We have the following estimates

$$
\begin{aligned}
\int_{s}^{s+0.5}|0.2 \cos (u)|+0.05(1+\sin (u)) d u & \leq 0.15 \\
\int_{s}^{s+\cos ^{2}(s)}|0.2 \cos (u)|+0.05(1+\sin (u)) d u & \leq 0.3 .
\end{aligned}
$$
Hence
$\sup _{t \geq 0} \int_{0}^{t} e^{-\int_{s}^{t} 0.2 \cos (u)+0.05(1+\sin (u)) d u}(0.15|0.2 \cos (s)|+0.015(1+\sin (s))) d s$
$<0.2001<1$.

It is easy to see that all the conditions of Theorem 4 hold for $\alpha=$ $0.2001<1$. Thus Theorem 4 implies that the solution $x$ of (3.5) converges to zero.

The first theorem provide sufficient conditions for convergence of the solution to zero. Let us now give a necessary condition for convergence of the solution to zero.

Theorem 5. Assume that (3) and the following condition hold

$$
\lim _{t \rightarrow \infty} \inf \int_{t_{0}}^{t} \frac{1}{\mu(\tau)} \log (1+\mu(\tau) D(\tau)) \Delta \tau>-\infty
$$

If all the solutions of (1.1) converge to zero, then (3.3) holds. 
Proof. Suppose that (3.3) fails. As (3.6) holds, this implies that

$$
K:=\sup _{t \geq t_{0}} e_{\ominus D}\left(t, t_{0}\right)<\infty,
$$

and that there exists a sequence $\left\{t_{n}\right\}$ with $t_{n} \rightarrow \infty$ as $n \rightarrow \infty$ such that the sequence $\left\{\int_{t_{0}}^{t_{n}} \frac{1}{\mu(\tau)} \log (1+\mu(\tau) D(\tau)) \Delta \tau\right\}_{n \geq 1}$ converges to a finite constant. So, we can choose a positive constant $H$ satisfying

$$
-H \leq \int_{t_{0}}^{t_{n}} \frac{1}{\mu(\tau)} \log (1+\mu(\tau) D(\tau)) \Delta \tau \leq H, \forall n \geq 1 .
$$

For the convenience of the statement, we put

$$
\begin{gathered}
g(s):=|a(s)| \int_{\sigma(s)}^{\sigma(s+h(s))}(|a(u)|+|b(u)|) \Delta u \\
+|b(s)| \int_{\sigma(s)}^{\sigma(s+h(s))}(|a(u)|+|b(u)|) \Delta u .
\end{gathered}
$$

Then, it follows from (3) that

$$
\int_{t_{0}}^{t_{n}} e_{D}\left(s, t_{0}\right) g(s) \Delta s \leq \alpha e_{D}\left(t_{n}, t_{0}\right)<e^{H}, \forall n>1 .
$$

The sequence $A_{n}:=\left\{\int_{t_{0}}^{t_{n}} e_{D}\left(s, t_{0}\right) g(s) \Delta s\right\}_{n \geq 1}$ is bounded, so it has a convergent subsequence. For brevity in notation, we can assume that $\lim _{t \rightarrow \infty} A_{n}=l$ for some $l$. Consequently, for any $\varepsilon_{0}>0$, there exists $n_{0} \geq 1$ such that

$$
\int_{t_{n_{0}}}^{t_{n}} e_{D}\left(s, t_{0}\right) g(s) \Delta s<\frac{\varepsilon_{0}}{2 K}
$$

for $K$ as in (3.7).

We replace $x_{0}$ by $\varepsilon_{0}$ and $t_{0}$ by $t_{n_{0}}$ in equation (3) to get the following equation

$$
\begin{aligned}
& \mathrm{x}(t)=\varepsilon_{0} e_{\ominus D}\left(t, t_{n_{0}}\right)+\int_{t_{n_{0}}}^{t} e_{\ominus D}(t, s) a(s)\left(\int_{\sigma(s)}^{\sigma(s+h(s))} E_{x}(u) \Delta u\right) \Delta s \\
& +\int_{t_{n_{0}}}^{t} e_{\ominus D}(t, s) b(s)\left(\int_{\sigma(s)}^{\sigma(s+r(s))} E_{x}(u) \Delta u\right) \Delta s, t \geq t_{n_{0}} .
\end{aligned}
$$

From lemma 3 , we known that the unique solution $\bar{x}$ of (3) is also a solution of (1.1) on $\left[t_{n_{0}}, \infty\right) \cap \mathbf{T}$. Using the relation

$x\left(t_{n_{0}}\right)-x(t)+\int_{t}^{t_{n_{0}}} a(s) x^{\sigma}(s+h(s)) \Delta s+\int_{t}^{t_{n_{0}}} b(s) x^{\sigma}(s+r(s)) \Delta s=0$, 
we can construct a solution $x$ of $(1.1)$ on $\left[t_{0}, \infty\right) \cap \mathbf{T}$ with $x(t)=\bar{x}(t)$, $t \in\left[t_{n_{0}}, \infty\right) \cap \mathbf{T}$. Because all solutions of (1.1) converge to zero, we have

$$
\lim _{t \rightarrow \infty} \bar{x}(t)=0 .
$$

We now fixe an $\varepsilon_{0}>0$ such that $\varepsilon_{0}<\frac{1-\alpha}{K e^{H}}$. From equation (3), we can obtain

$$
\begin{gathered}
\sup _{t \geq t_{n_{0}}}|\bar{x}(t)| \leq K\left|\varepsilon_{0}\right| e_{D}\left(t_{n_{0}}, t_{0}\right)+\alpha \sup _{t \geq t_{n_{0}}}|\bar{x}(t)| \\
\leq K\left|\varepsilon_{0}\right| e^{H}+\alpha \sup _{t \geq t_{n_{0}}}|\bar{x}(t)|
\end{gathered}
$$

which yields

$$
\sup _{t \geq t_{n_{0}}}|\bar{x}(t)| \leq \frac{K e^{H} \varepsilon_{0}}{1-\alpha}<1 .
$$

Hence, an application of the inequality $a+b+c \geq|a|-|b|-|c|$ to (3) gives us

$$
\bar{x}\left(t_{n}\right) \geq \varepsilon_{0} e_{\ominus D}\left(t_{n}, t_{n_{0}}\right)-\int_{t_{n_{0}}}^{t_{n}} e_{\ominus D}\left(t_{n}, s\right) g(s) \Delta s, n \geq n_{0} .
$$

This combine with (3.9) and (3.8) implies that

$$
\begin{aligned}
& \bar{x}\left(t_{n}\right) \geq e_{\ominus D}\left(t_{n}, t_{n_{0}}\right)\left(\varepsilon_{0}-e_{\ominus D}\left(t_{n_{0}}, t_{0}\right) \int_{t_{n_{0}}}^{t_{n}} e_{D}\left(s, t_{0}\right) g(s) \Delta s\right) \\
\geq & e_{\ominus D}\left(t_{n}, t_{n_{0}}\right)\left(\varepsilon_{0}-K \int_{t_{n_{0}}}^{t_{n}} e_{D}\left(s, t_{0}\right) g(s) \Delta s\right) \\
\geq & e_{\ominus D}\left(t_{n}, t_{n_{0}}\right)\left(\varepsilon_{0}-K \frac{\varepsilon_{0}}{2 K}\right) \geq \frac{\varepsilon_{0} e^{-2 H}}{2},
\end{aligned}
$$

which contradicts with (3.10). The proof is completed.

Our results can be extended to the following general advanced dynamic equation with several terms

$$
x^{\triangle}(t)+\sum_{k=1}^{N} a_{k}(t) x^{\sigma}\left(t+h_{k}(t)\right), t \in\left[t_{0}, \infty\right) \cap \mathbf{T},
$$

where $a_{k}$ and $h_{k}$ are rd-continuous functions and $h_{k}(t) \geq 0$.

Indeed, as in Lemma 3, we can rewrite (3.11) as follows

$$
\begin{gathered}
x(t)=x_{0} e_{\ominus \bar{D}}\left(t, t_{0}\right) \\
+\int_{t_{0}}^{t} e_{\ominus \bar{D}}(t, s) \sum_{k=1}^{N}\left(a_{k}(s) \int_{\sigma(s)}^{\sigma\left(s+h_{k}(s)\right)} \bar{E}_{x}(u) \Delta u\right) \Delta s, t \in\left[t_{0}, \infty\right) \cap \mathbf{T},
\end{gathered}
$$


where $\bar{D}(t)=\sum_{k=1}^{N} a_{k}(t)$ and $\bar{E}_{x}(t)=\sum_{k=1}^{N} a_{k}(t) x^{\sigma}\left(t+h_{k}(t)\right)$. Then, we can get the following theorem without new difficulties.

Theorem 6. Suppose that the following condition holds,

$$
\sup _{t \geq t_{0}} \int_{t_{0}}^{t} e_{\ominus \bar{D}}(t, s) \sum_{k=1}^{N}\left(\left|a_{k}(s)\right| \int_{\sigma(s)}^{\sigma\left(s+h_{k}(s)\right)} \sum_{i=1}^{N}\left|a_{i}(u)\right| \Delta u\right) \Delta s:=\alpha<1 .
$$

If

$$
\bar{D} \in \mathcal{R}^{+}, \lim _{t \rightarrow \infty} \lim \int_{t_{0}}^{t} \frac{1}{\mu(\tau)} \log (1+\mu(\tau) \bar{D}(\tau)) \Delta \tau=\infty,
$$

then any solution $x$ of (3.11) converges to zero.

Acknowledgements. The authors would like to thank the anonymous referee for his valuable comments.

\section{References}

[1] M. Adıvar, Y. N. Raffoul, Existence of periodic solutions in totally nonlinear delay dynamic equations. Electronic Journal of Qualitative Theory of Differential Equations 2009, 1, pp. 1-20, (2009).

[2] A. Ardjouni, I. Derrardjia and A. Djoudi, Stability in totally nonlinear neutral differential equations with variable delay, Acta Math. Univ. Comenianae, Vol. LXXXIII, 1, pp. 119-134, (2014).

[3] A. Ardjouni, A Djoudi, Existence of periodic solutions for nonlinear neutral dynamic equations with functional delay on a time scale, Acta Univ. Palacki. Olomnc., Fac. rer. nat., Mathematica 52, 1, pp. 5-19, (2013).

[4] A. Ardjouni, A Djoudi, Stability in neutral nonlinear dynamic equations on time scale with unbounded delay, Stud. Univ. Babeç-Bolyai Math. 57, No. 4, pp. 481-496, (2012).

[5] A. Ardjouni, A Djoudi, Fixed points and stability in linear neutral differential equations with variable delays, Nonlinear Analysis 74, pp. 2062-2070, (2011). 
[6] M. Bohner, A. Peterson, Dynamic Equations on Time Scales, An Introduction with Applications, Birkhauser, Boston, (2001).

[7] M. Bohner, A. Peterson, Advances in Dynamic Equations on Time Scales, Birkhäuser, Boston, (2003).

[8] T. A. Burton, Liapunov functionals, fixed points and stability by Krasnoselskii's theorem, Nonlinear Stud. 9, pp. 181-190, (2001).

[9] T. A. Burton, Stability by fixed point theory or Liapunov theory: A Comparaison, Fixed Point Theory, 4, pp. 15-32, (2003).

[10] T. A. Burton, Stability by Fixed Point Theory for Functional Differential Equations, Dover Publications, New York, (2006).

[11] N. T. Dung, Asymptotic behavior of linear advanced differential equations, Acta Mathematica Scientia, 35B (3): pp. 610-618, (2015).

[12] I. Derrardjia, A. Ardjouni and A. Djoudi, Stability by Krasnoselskii's theorem in totally nonlinear neutral differential equations, Opuscula Math. 33 (2), pp. 255-272, (2013).

[13] S. Hilger, Ein Maßkettenkalkül mit Anwendung auf Zentrumsmannigfaltigkeiten, Ph. D. thesis, Universität Würzburg, Würzburg, (1988).

[14] E. R. Kaufmann, Y. N. Raffoul, Stability in neutral nonlinear dynamic equations on a time scale with functional delay, Dynamic Systems and Applications 16, pp. 561-570, (2007).

[15] D. R. Smart, Fixed point theorems, Cambridge Tracts in Mathematics, no. 66, Cambridge University Press, London-New York, (1974).

\author{
Malik Belaid \\ Applied Mathematics Lab, \\ Faculty of Sciences, \\ Department of Mathematics, \\ University of Annaba, \\ P. O. Box 12, Annaba 23000, \\ Algeria \\ e-mail: malikbelaid51@gmail.com
}




\author{
Abdelouaheb Ardjouni \\ Department of Mathematics and Informatics, \\ University of Souk Ahras, \\ P. O. Box 1553, Souk Ahras 41000, \\ Algeria \\ e-mail: abd_ardjouni@yahoo.fr \\ and
}

\author{
Ahcene Djoudi \\ Department of Mathematics, \\ Applied Mathematics Lab, \\ Faculty of Sciences, \\ University of Annaba, \\ P. O. Box 12, Annaba 23000, \\ Algeria \\ e-mail: adjoudi@yahoo.com
}

